\title{
18
}

\section{Surges Associated with Filling of Stormwater Storage Tunnels}

\author{
Steven J. Wright, Jose G. Vasconcelos Neto and Karen E. Ridgway
}

Surges may form during rapid filling incidents in stormwater storage tunnels as the tunnel undergoes a transition between a free surface flow and a surcharged state. The current state of knowledge does not allow specification of conditions that lead to extreme surges, that would provide the basis for design of surge relief systems. A laboratory model was developed to provide insight into the geometrical and flow conditions that will develop large surges. The model was tested at a variety of slopes and for flow conditions that allow for the investigation of the influence of the hydrograph at the inflow points into the tunnel. Experimental findings indicate that the largest surges occurred when a hydraulic bore just formed as it approached the surge relief structure. The maximum surges also occurred when the tunnel slopes downwards from the surge chamber. It was also observed that in conditions where the exhaust of air is impeded from the system, the compression of air may induce motion due to the air pressure acting on the water surface. Current numerical models developed to describe the surge process have not included this effect in their formulation.

\subsection{Introduction}

Below grade storage tunnels may be used to reduce the frequency and magnitude of environmental discharges of urban stormwater or combined sewage flows. Operational problems have been experienced in constructed

\footnotetext{
Wright, S.J., J. Vasconcelos and K. Ridgway. 2003. "Surges Associated with Filling of Stormwater Storage Tunnels." Journal of Water Management Modeling R215-18. doi:

10.14796/JWMM.R215-18.

(C) CHI 2003 www.chijournal.org ISSN: 2292-6062 (Formerly in Practical Modeling of Urban Water Systems. ISBN: 0-9683681-7-4)
} 
systems with "geysering" or surface discharges of air-water mixtures through manholes or other vertical shafts due to surges apparently initiated during the transition from free surface flow to a surcharged state in the tunnel. Previous investigations into these flows have primarily relied on numerical modeling due to the system complexity (Guo and Song, 1990, 1991; Li and McCorquodale, 1999). Limited experimental studies such as Cardle et al., (1989) and Li and McCorquodale (1999) have been conducted primarily for the purpose of obtaining data used for model calibration/verification and the conditions that may be expected to lead to large surges remain poorly understood. This is a critical issue with respect to design since the design rainfall event may not necessarily result in the highest magnitude of surge within a given system as a critical parameter may be the rate of inflow at the time the tunnel goes full, for example. In an attempt to gain some insight into the role of various system parameters on the surge magnitude, a laboratory model was constructed to mimic the essential features of a proposed design for a flow-through storage tunnel proposed for the City of Dearborn, Michigan. An investigation into the role of various system parameters on the observed magnitude of surges is being conducted. This chapter describes the proposed system design and the features of the laboratory model to capture the essential features of the system while still allowing a relatively straightforward model setup. Preliminary results of the testing are presented along with tentative conclusions as to the design issues that are considered to be most important in influencing surges in the system.

\subsection{System Description}

A conceptual design for a flow-through storage tunnel system has been developed for the City of Dearborn, Michigan (NTH, 2000). The tunnel has a proposed length of $9500 \mathrm{~m}$ and an inside diameter of $5.2 \mathrm{~m}$. Inflow into the tunnel is through a series of 15 dropshafts at the locations where combined sewer overflows formerly discharged to the Rouge River. The tunnel has been sized to accommodate a $10-y$ rainfall event and has a design capacity of approximately $175 \mathrm{~m}^{3} / \mathrm{s}$. In order to work within head constraints, there are to be three overflow points distributed at roughly $3000 \mathrm{~m}$ intervals along the tunnel. So long as the system storage volume is not exceeded, the system behaves as a single storage element. At high system discharges, gates isolating the three tunnel segments are closed and overflows to the Rouge River would occur once the storage capacity has been exceeded in each segment. The preliminary design is for discharges by gravity in the two upstream segments 


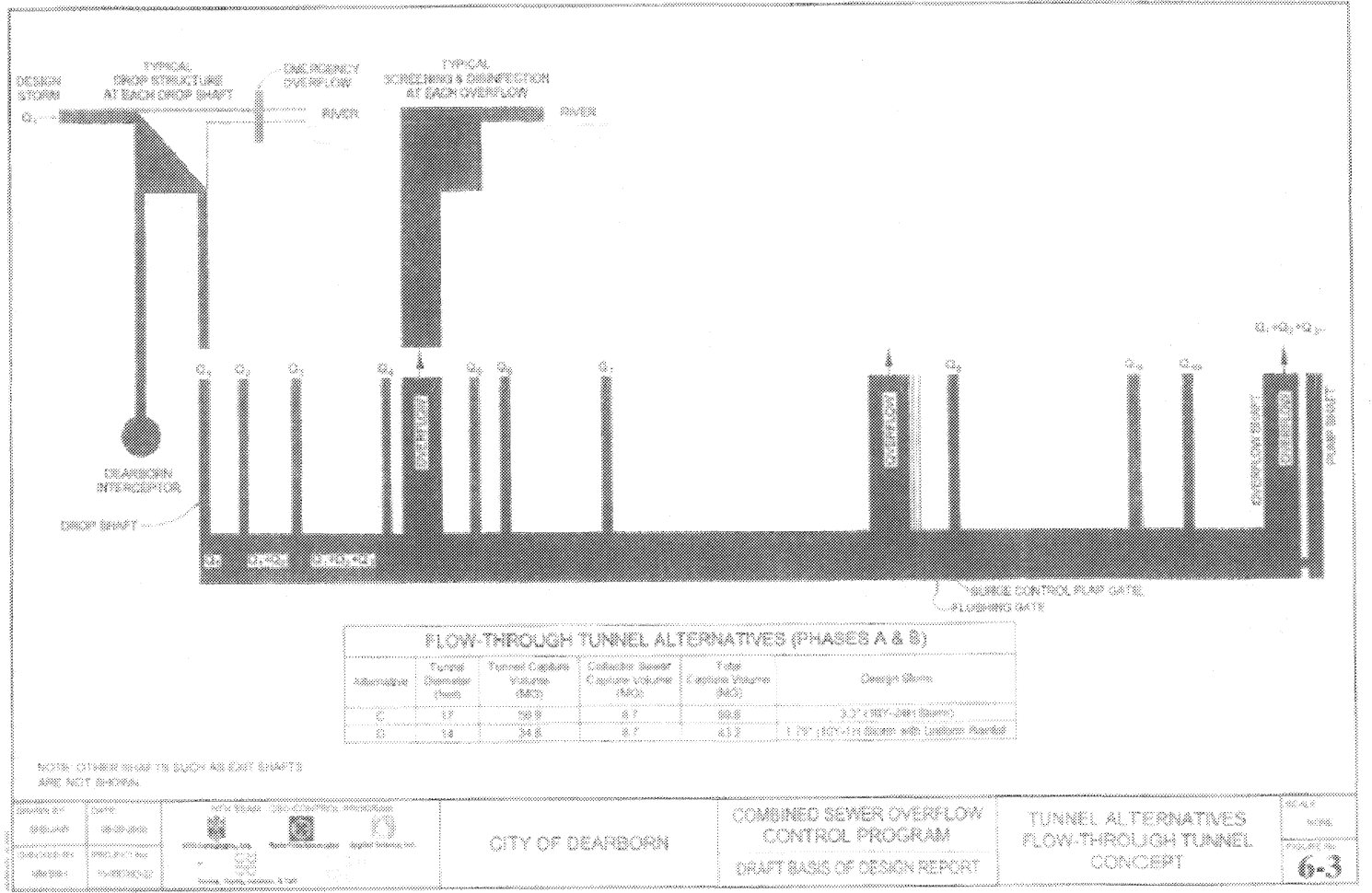

Figure 18.1 Schematic of proposed Dearborn flow-through tunnel system. 
while a pumping station will be required at the end of the downstream segment. Figure 18.1 is a schematic of the proposed tunnel system. Large diameter vertical riser shafts located at the junction between tunnel segments will be split vertically to allow the isolation between segments. The vertical wall will contain a lower gate structure for isolating the tunnel segments and an upper flap gate structure for surge relief for the downstream tunnel segment. A surge chamber would be provided at the far upstream end of the system to provide relief for surges that are anticipated to occur when tunnel filling occurs.

\subsection{Background}

Several previous storage tunnel systems have experienced a phenomenon referred to as geysering after being placed in operation. Geysering is manifested by a combination of air and water blowing out to grade through manholes. The Chicago deep storage tunnel project experienced a number of such incidents (Guo and Song, 1990, 1991). The exact sequence of events in a geysering incident is not totally clear, but is related to the occurrence of the transition between free surface and pressurized flow within the tunnel as it transitions to a filled state. Since there are multiple inflow points, the possibility exists for the entrapment of air at the tunnel crown if the tunnel fills rapidly. The geysering may simply be a release of this air as it is transported laterally through the tunnel to relief points at manholes or other vertical shafts that are already in a surcharged state. The migration direction of the air depends on the tunnel slope as well as the filling sequence. Regardless of the presence of entrapped air, a surge will develop in a nearly horizontal tunnel as it undergoes the transition between free surface and pressurized flow. Therefore adequate venting of the air within the system may not provide relief from environmental releases of stormwater to grade.

Surge control was implemented in the Chicago system, for example, by throttling the inflows into the storage tunnel as it approaches the filled state. By reducing the fill rate as the tunnel went through the transition to a pressurized state, the magnitude of surges was reduced to an acceptable level, effectively eliminating the geysering problem. However, this surge control alternative is not viable for the Dearborn flow through system. Current state regulations require chlorination of the combined sewage in the tunnel with fifteen minutes of contact time prior to any environmental release. This requirement effectively constrains the discharge points to the three release points at the ends of the three tunnel segments. Consequently it is not possible to throttle the inflows at the 
various inflow dropshaft since this would constitute an untreated release at each location. Adequate surge relief must be designed into the system, so it is important to understand the conditions that lead to large surges and/or geysering in the system. Analysis based on runoff from a design rainfall evenly distributed over a watershed may not necessarily prescribe the worst conditions with respect to surging in a system. For example, it may occur that preferential filling at one end of a system leads to higher surges at the opposite end. Also, filling from multiple inflow points may exacerbate the entrapment of air at the tunnel crown as it fills and the subsequent release of this trapped air at intermediate manholes may be more violent than if the tunnel filled uniformly from one end to the other.

Existing numerical models for transient flow do not apparently shed much light on these issues. Running SWMM for the high flows associated with the design rainfall event yielded results that did not appear to be physically consistent. As the tunnel became surcharged, high hydraulic grade line spikes appeared at internal segments in the tunnel. We interpret this to be related to the fact that SWMM does not accurately represent bores that could develop in a rapidly filing tunnel. The model code apparently artificially fixes the bore speed by the model spatial and temporal discretization. Transient pressure reflections off the physically. inconsistent bore result in unrealistic computations of hydraulic grade line elevations in the surcharged portion of the system.

Other models have been developed to more realistically simulate the surges that would occur on rapid tunnel filling. However, these models have other deficiencies. For example, some model formulations use a "Preisman slot" (e.g. Cunge and Holly, 1981). This slot is an artificial device used in the model to compute a gravity wave speed in the tunnel/slot combination that matches the pressure wave speed in a closed conduit. Although this approach would avoid at least some of the difficulties observed in the SWMM simulations, there is still a difficulty with the presence of trapped air. Air disappears from the system through the slot and any effects of the air motion or pressurization on the dynamics of the water flow cannot be explicitly accounted for.

Based on these concerns, it was decided to construct a physical model that reproduced the essential elements of the Dearborn flow through tunnel system. Similar to other previous laboratory investigations, it was intended to collect data for numerical model calibration/verification. However, an additional objective was to systematically vary several system variables that were assumed to be critical to surge behavior in an attempt to gain some insight into the conditions that might lead to peak system surges and/or geysering. 


\subsection{Physical Model}

It was generally not possible to reproduce the entire tunnel length at a reasonable scale in a laboratory model. Therefore the decision was made to model only a tunnel length that would be consistent with the distance between an inflow dropshaft and a surge shaft; this distance would be on the order of $750 \mathrm{~m}$ prototype. Because of other constraints described below, a $15 \mathrm{~m}$ length in the laboratory would require a 1:50 model scale in order to reproduce this prototype length. The required model diameter is then approximately $100 \mathrm{~mm}$ to reproduce the $5.2 \mathrm{~m}$ diameter tunnel. Clear acrylic tubing was used in the model construction in order to be able to observe the movement of bores and trapped air within the tunnel. In order to be able to investigate the effect of tunnel grade on the magnitude of surges, the tunnel model was installed in a tilting flume. By adjusting the flume slope, the tunnel slope could be easily modified without concern for other alignment changes. The model was therefore carefully leveled to provide a horizontal alignment as observed by an air bubble at the top of the nearly full pipe, and all other sloping pipe configurations were created by adjusting the flume slope. The acrylic pipe is manufactured in $2 \mathrm{~m}$ long segments and is joined by a PVC sleeve at each joint. The intention is to use these joints to install air relief vents and other similar transitions when more complex tunnel configurations are to be studied.

All three tunnel segments are conceptually similar in that they provide for an overflow at approximately a constant elevation at the downstream end although the details vary in the prototype. This effect was modeled by connecting the acrylic pipe to a storage box of limited height, essentially modeling the overflow elevation at the downstream end. Model operation consists of filling the pipe through a constant rate inflow through the fill box. The purpose of this arrangement is to simulate a nearly constant downstream head such as would be achieved with the downstream pump station or intermediate overflow discharges. It was also considered that if the model was filled through an intermediate riser located along the pipeline, there would be a lower surge than filling at the overflow location from where the resulting transient propagates only in one direction. Therefore, both the inflow and outflow occur at the fill box while the only motion in the remainder of the system is the surge behavior associated with changing from an initially stagnant condition to a new steady state where the inflow matches the flow spilling over the weir at the fill box. Subsequent investigations will examine multiple inflow points to investigate the effect on surge reduction. A vertical riser constructed of larger diameter clear acrylic pipe was installed at the opposite end of the 
system to mimic the effects of surge relief being installed there. The details of this surge relief (diameter of the riser) will affect the magnitude of the surge but a numerical model will be able to simulate any surge relief installed at this location. The height of the surge riser is much greater than the top of the overflow box and selected to contain any surges created in the experiments.

A major issue was how to deal with inflow conditions. In the prototype, inflow hydrographs at each dropshaft have be determined with a rainfall/runoff model and these are used as inputs into a numerical model. Modeling this sort of inflow distribution would be extremely difficult in the laboratory. It was eventually decided to simply input water at a constant flow rate representative of the maximum discharge in the inflow hydrograph. In order to simulate the effect of the low flows early on the rising hydrograph, the inflow was started with the laboratory system in a partially full state. Therefore, the laboratory variables of constant inflow rate and initial water level in the system can be thought of as representing different inflow hydrographs in a crude fashion. A two-way valve is installed in the discharge line for the inflow into the system. Flow is initially established by using the valve in the diverted setting so that no flow is entering the model. Once the metered discharge is in a steady condition, it is quickly switched into the model and the experiment performed. Entrainment of air into the inflow has been minimized although this is also a potentially important variable to be investigated later.

A schematic of the system is indicated in Figure 18.2. The pipe is $14.6 \mathrm{~m}$ long and approximately $100 \mathrm{~mm}$ diameter. The riser pipe is $190 \mathrm{~mm}$ diameter while the fill box has a cross-section $250 \mathrm{~mm}$ on a side and an overflow level about $250 \mathrm{~mm}$ above the pipe invert. In order to avoid confusion of what is meant by upstream and downstream (the system is filled from the downstream end of the prototype system), the two ends of the model are referred to as the fill end and the riser end. Vertical rulers are used to provide a scale at each end of the system. Data is collected at the riser end of the model with a digital video

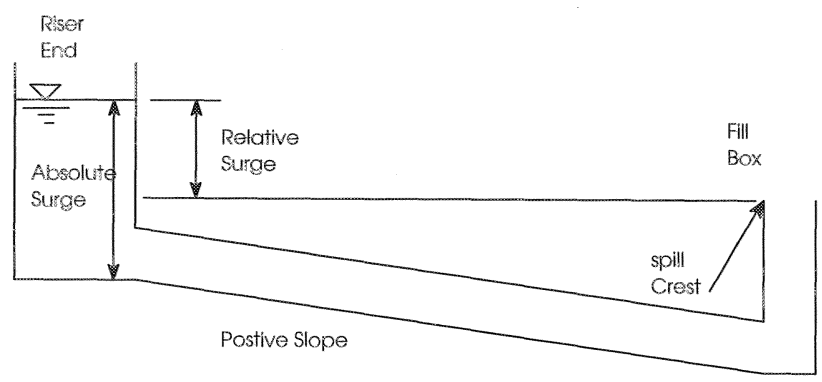

Figure 18.2 Schematic of physical model. 
camera and at the fill end of the system with a standard VHS format video camera. Digital information at the riser end of the pipe is later downloaded into a computer and can be analyzed on a frame-by-frame basis to determine exact timing (relative to the commencement of inflow) and magnitude of the water surface variation in the riser at the end of the system. The surge reported below is what we refer to as the relative surge, i.e. the surge relative to the overflow level in the fill box. This is considered to be the most appropriate measure since this quantity would go to zero in the limit of a very slow fill rate. Future plans include the installation of pressure transducers along the pipeline in order to observe pressure variations during the transient.

\subsection{System Variables}

The preliminary investigation has concentrated on the effect of several variables that were felt to be potentially significant with respect to the surge. These include both the fill rate and the initial water level in the model as indicative of the inflow hydrographs in the system described above. Another variable was the slope of the pipe. Pipe slopes investigated included horizontal and both positive and adverse (in which the pipe slopes upwards from the riser to the fill box) slopes of 0.1 and $0.2 \%$. The pipe slope was considered to be important with respect to both the dynamics of the hydraulic bores propagating through the system as well as the dynamics of air pockets in the system. The initial system was constructed without providing for air venting along the length of the pipe. Subsequent experiments will include vents at various locations to determine the influence on observed surges. Future plans also include varying the amount of air entrained into the inflow, filling at intermediate locations along the pipe, and filling at multiple inflow points. The initial experiments are intended to gain some insight as to the processes that most influence the occurrence of large surges in a filling stormwater storage tunnel, with a secondary goal to collect experimental data that can be used in numerical model validation.

\subsection{Experimental Results}

\subsection{Influence of Model Scale}

One outcome that was not initially anticipated was that the Reynolds number for flow in the pipe is sufficiently low and there is an issue as to whether the flow is turbulent or not. Although Reynolds numbers in the pipe based on the 
inflow rate would be above the turbulent transition, the bulk of the inflow does not flow through the pipe and the oscillations in the surge guarantee that the flow will be below the laminar threshold (considered to be a Reynolds number of 2000 for the purpose of the subsequent modeling) during at least a portion of the oscillations. In order to investigate the influence of this effect, some preliminary runs were made with the model pipe initially filled to just above the crown. In this situation, a simple surge model can be developed to describe the oscillations in the system. Details of the model are omitted here, but it is based on the coupling of a one-dimensional incompressible momentum equation within the pipe with appropriate continuity equations at both the fill box and the surge riser. An experiment in which all simulated Reynolds numbers were less than 2000 is presented in Figure 18.3 along with the computed results.

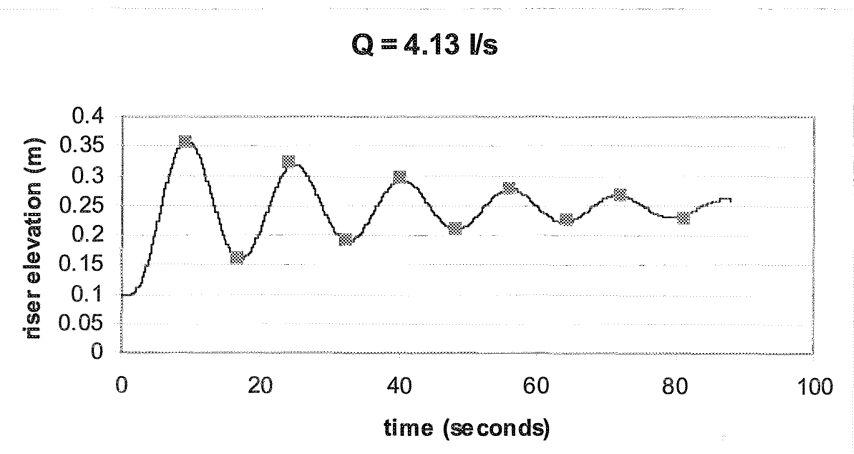

Figure 18.3 Comparison of observed levels in riser and model predictions with laminar friction.

Data presented are the observed maximum and minimum levels in the surge riser along with the times of their occurrence. The model quite accurately captures both the damping of the surge peaks and the timing of them. In order to investigate the effect of the laminar flow on the observations, the model was re-run with a constant friction factor of 0.04 , a value that would be representative for turbulent flow in a smooth pipe just above the transition Reynolds number. The results for this simulation are presented in Figure 18.4 and it can be seen that the first surge peak, as well as the timing, is still described fairly well with only approximately a one percent increase (compared to the laminar flow simulation) in the initial peak surge relative to the overflow level. The damping is computed to be less than the observed, but the differences are 
relatively minor. Therefore, it is assumed that the initial surge peaks that are presented in the results to follow are representative of those that would occur in a prototype system of much larger scale.

\section{$Q=4.13 \mathrm{l} / \mathrm{s}$}

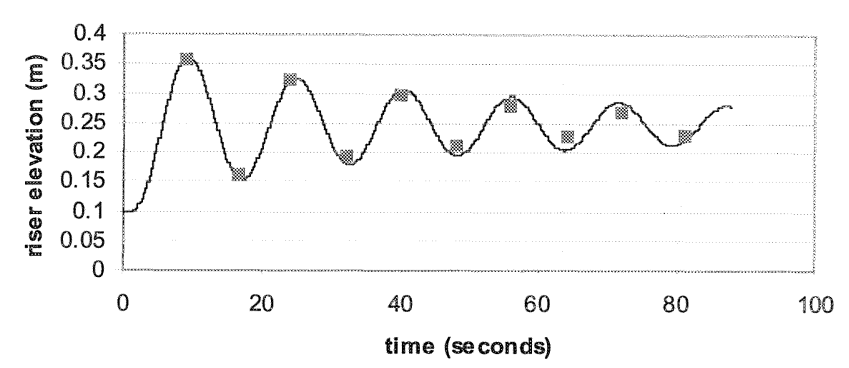

Figure 18.4 Comparison of observed levels in riser and model predictions with constant turbulent friction factor.

\subsubsection{Effect of Inflow Hydrograph}

It is fairly intuitive that an increase in the fill rate will result in an increased surge height in the riser shaft under the conditions of constant inflow rate. However, the situation is much more complex when one considers the effect of a variable inflow rate. Very little guidance is available in the literature in order to understand the conditions that will result in maximum system surge. Guo and Song (1990) make only oblique references to different combinations of flow conditions that can result in large surges. Given the lack of understanding of the circumstances that can lead to high surges, an initial experimental program was conducted with a horizontal pipe at three different discharges and with different initial water levels. Example results at a single discharge are presented in Figure 18.5 which shows that there are surge maxima at intermediate initial water levels for a given inflow rate. The explanation for this was clear from direct observations of the physical nature of the flow. At small initial water levels, a bore of small height formed, not filling the entire pipe. This bore propagated to the riser and reflected back into the tunnel with an increased height. Depending on the initial water level and flow rate, this reflected bore may or may not fill the pipe but in either case possesses some inertia back towards the fill end of the pipe. When the pipe eventually completely filled, air tended to be trapped in the system and would 


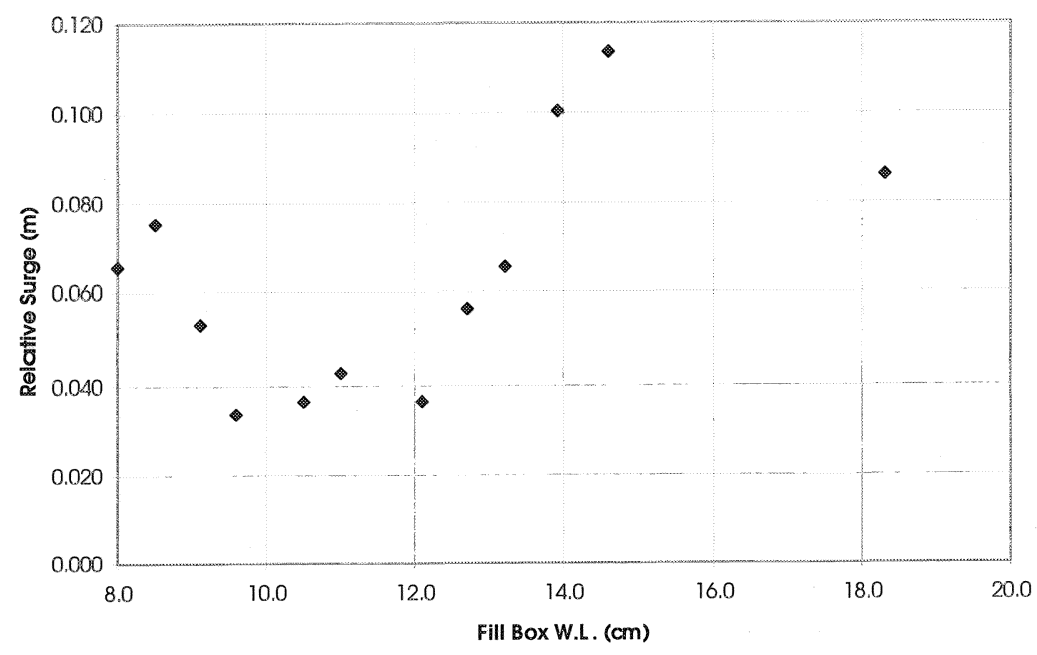

Figure 18.5 Variation of relative riser surge with initial water level in tunnel $(\mathrm{Q}=2.13 \mathrm{l} / \mathrm{s}$, horizontal tunnel alignment).

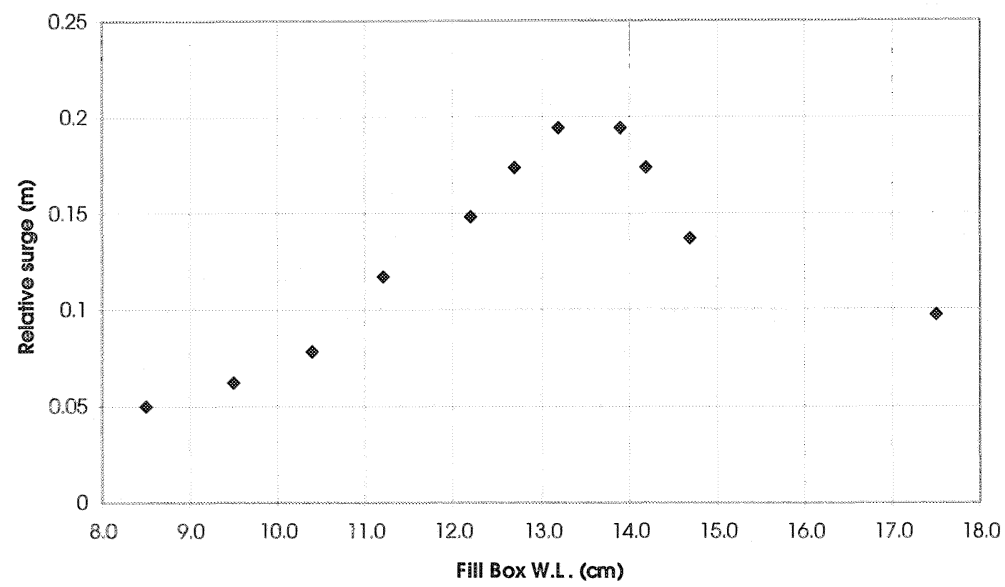

Figure 18.6 Variation of relative riser surge with initial water level in tunnel $(\mathrm{Q}=3.06 \mathrm{l} / \mathrm{s}$, horizontal tunnel alignment).

blow out either or both ends of the pipe during subsequent oscillations. The maximum surge height occurred approximately when the initial bore just filled the pipe at the riser end. This is a reasonable outcome since the inertia in the bore is directed towards the riser at the instant that the riser starts to rapidly fill. The 
secondary lower peak in Figure 18.5 at a lower initial water level is related to a similar phenomenon although in that situation, it is the second reflection that is synchronized with the rapid filling of the riser. Figure 18.6 depicts a similar set of data except at a different fill rate. In this case, the maximum surge occurs at a different initial water level since the size of the initial bore is dependent on the fill rate. However, the maximum surge still occurs at roughly the condition where that surge just fills the tunnel as it approaches the riser end of the system. In the laboratory investigation, the initial bore formed near the fill end of the tunnel. However, in the prototype situation with a rising hydrograph, an increasing discharge will cause a bore to form if the tunnel is sufficiently long and the rate of fill is sufficiently rapid (Henderson, 1966). Therefore, it is reasonable to expect similar behavior in long storage tunnel systems. A difficulty is that it would not be possible to determine the conditions necessary for the creation of a maximum surge without application of a numerical analysis that accurately computes the bore formation and propagation. This is one of the objectives of the current research.

\subsubsection{Effect of Tunnel Slope}

The situation becomes more complex when the tunnel slope is not horizontal. For a horizontal tunnel, there is essentially no air trapped in the system when the maximum surge occurs as the initial bore filling the tunnel forces the air from the system through the riser. It appears that venting of the system would have little impact on the magnitude of the maximum surge under those circumstances. Once initial results were obtained with the horizontal tunnel, an attempt was made to anticipate the effect of tunnel slope on the magnitude of the maximum surge. It was reasoned that the case of positive slope (drop in tunnel elevation between the riser and the fill end) should represent a worst-case situation with respect to surge. The reasoning behind this considered that the maximum surge would still occur when the bore just filled the tunnel at the riser end. However, the greater depths at the fill end at this condition would yield a more rapidly propagating bore in that portion of the tunnel potentially increasing the surge magnitude due to the higher inertia in the tunnel length.

This reasoning was consistent with observations as indicated in Figure 18.7. Fairly similar system behavior was observed except that the maximum surge height was reached more quickly in the riser due to the faster bore speeds towards the fill end of the system. The increases in surge height were fairly modest for the experiments performed at $0.2 \%$ slope, only on the order of $10 \%$. 


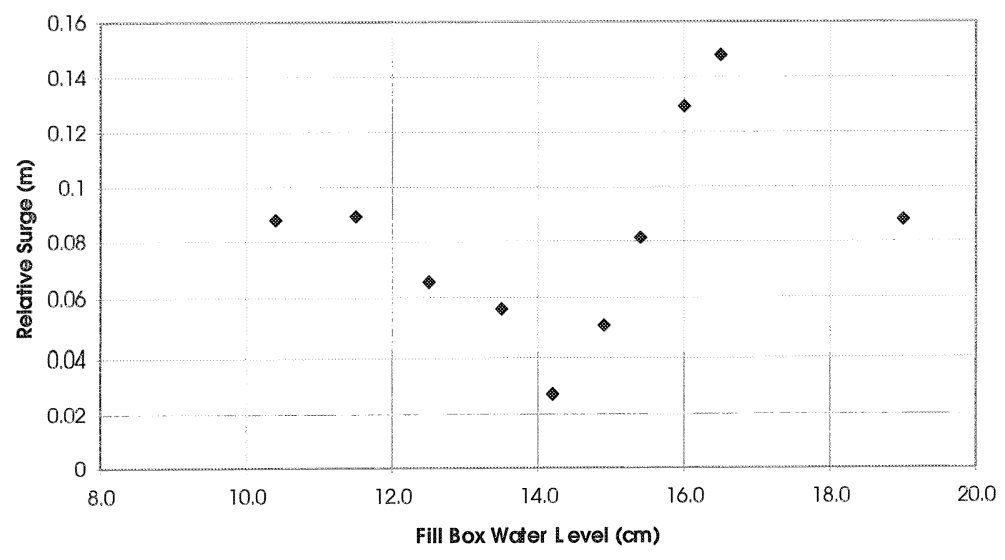

Figure 18.7 Variation of relative riser surge with initial water level in tunnel $(\mathrm{Q}=2.161 / \mathrm{s}, 0.2 \%$ positive slope $)$.

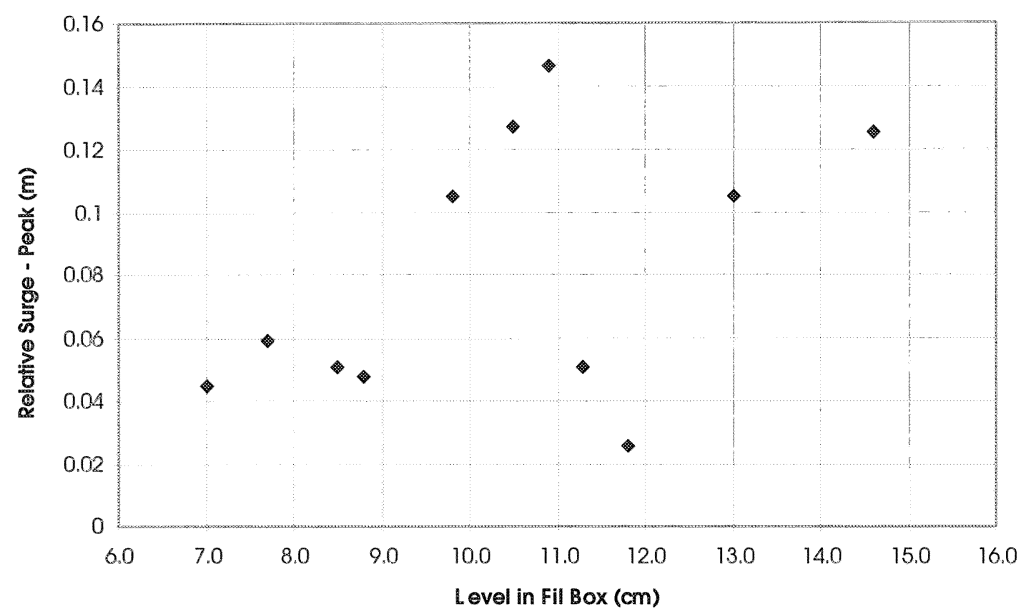

Figure 18.8 Variation of relative riser surge with initial water level in tunnel $(\mathrm{Q}=3.061 / \mathrm{s}, 0.2 \%$ adverse slope $)$.

The absolute surge levels in the riser increased significantly since the tunnel slope decreases the initial elevation at the riser relative to the overflow level compared to the horizontal tunnel situation. It is likely that the increases in relative surge due to the pipe slope would be greater if there were inadequate surge protection volume at the riser end. 
It was also observed that the maximum surges occurred at somewhat higher initial water levels than required to just fill the tunnel at the riser end. This may be due to the effects of surface tension in a relatively small experimental system since the bore completely filled the tunnel at the fill end at lower initial water levels and perhaps had to overcome surface tension to pull away from the tunnel crown as it propagated into the shallower depths towards the riser end of the system. This issue will require further investigation.

The situation was expected to be more complex under conditions of adverse slope where the initial water depths in the tunnel were greater at the riser end of the system than at the fill end. Figure 18.8 presents the results of the system behavior under these conditions; it was found that surge magnitudes (both relative and absolute) were decreased under these conditions. A major issue is that a bore propagating into deeper water may reach a tunnel filling condition only at the riser end of the system, trapping substantial volumes of air behind it. In the current experimental configuration, this air is only allowed to exit through either the fill box or the riser shaft and in general, it was through a combination of both as the subsequent oscillations in the tunnel carried the air back and forth through the system. It is likely that tunnel venting may have a significant effect on the surge behavior and it is planned to investigate this effect in the near future.

Figure 18.9 contains a direct comparison of the relative surge heights observed at the same flow rate for the three different slope configurations. It is seen that there are relatively small but consistent differences in the surge behavior with the highest surges at positive slopes and lowest under adverse slope conditions. It is expected that these differences would have been exacerbated had less riser storage been provided.

The flow behavior for adverse slopes was much more complex than observed with the horizontal and positive slopes due to air entrapment. Air entrapment did occur during experiments in those cases as well as at low initial water levels when the system did not attain its maximum surge. In the adverse slope case, air entrapment is significant at conditions of maximum surge. We observed a behavior that initially was difficult to understand. When the initial water level at the riser end of the tunnel is nearly at the crown, the water level in the riser begins to increase prior to the arrival of the bore and seals off the pipe crown causing large volumes of trapped air to be expelled ahead of the arriving bore. Since the bore, as a nonlinear wave, should propagate more rapidly than any smaller amplitude disturbances, the arrival of the bore should precede any other motion in the still fluid at the riser. Subsequent experiments have shown that the compression of air ahead of the bore is responsible for this 


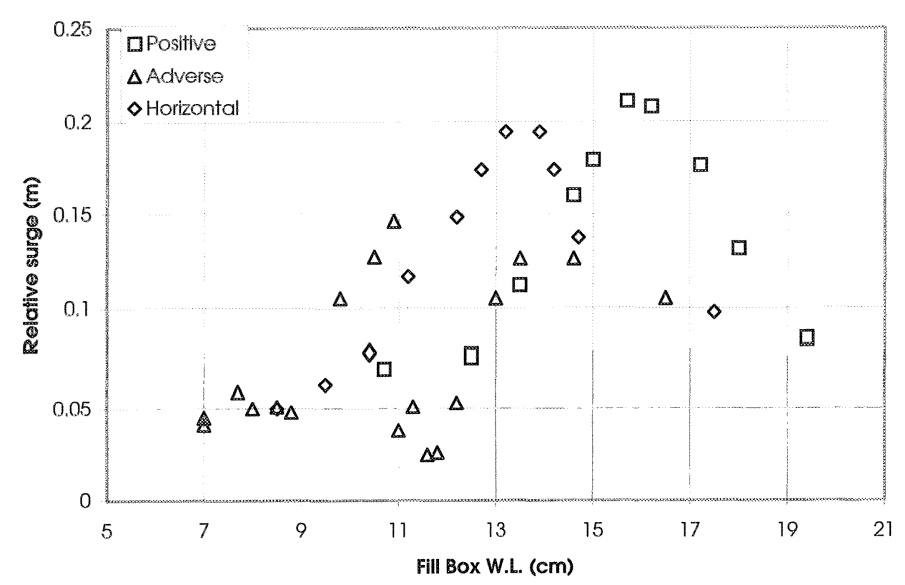

Figure 18.9 Comparison of relative surge for $Q=3.061 / \mathrm{s}$ and different slopes.

behavior, necessitating a more complex numerical model that simulates the dynamics of the air displacement in the system. We plan additional experiments to understand this behavior and to collect data for verifying simulations in which the compression of air trapped within the system has an influence on the surge behavior.

\subsection{Conclusions}

The experimental results presented can be considered to be preliminary results in a continuing investigation into the development of large magnitude surges in a filling stormwater storage tunnel system. This data will also be used to guide the formulation and verification of a numerical model that is capable of simulating the surge behavior in a prototype system and therefore can be used as a basis for design analysis of required surge chamber volumes or other system components to mitigate surging. Based on the findings to date, the following conclusions are offered:

- When the tunnel slope is horizontal or with an increasing invert elevation away from the filling location, the maximum surge occurs when a hydraulic bore forms that just fills the tunnel at the far end of the system. The conditions that will result in this will depend on the inflow hydrograph and it will be difficult to define worst-case conditions for design for even a simple system. 
- Under the above-noted conditions of horizontal or positive tunnel slope, trapped air does not play a role at conditions of maximum surge. In a real system with multiple inflow points, the validity of this conclusion remains to be demonstrated.

- For a case where the pipe slope causes the bore formed in a filling tunnel to propagate into greater initial water depths, the entrapment of air has a significant impact on the surge behavior and a numerical model will need to be capable of capturing this behavior in order to accurately simulate the surge.

- With regard to the effect of slope, highest surges occur in circumstances where the bore propagates up a sloping pipeline.

- In geometries in which the air could not be readily expelled, the compression of the air induces motion ahead of the free surface waves initiated by the inflow. This indicates the need for including the dynamic effect of the trapped air in any numerical model developed to simulate the surge process.

\section{References}

Cardle, J.A., C.C.S. Song and M. Yuan (1989), "Measurements of Transient Mixed Flow," Journal of Hydraulic Engineering, Vol. 115, No. 2, pp. 169-182

Cunge, J.A. and F.M. Holly, (1981), "Practical Aspects of Computational River Hydraulics", Pittman, Boston.

Guo, Q. and C.C.S. Song, (1990), "Surging in Urban Storm Drainage Systems," Journal of Hydraulic Engineering, Vol. 116, No. 12, pp. 1523-1537.

Guo, Q. and C.C.S. Song, (1991), "Dropshaft Hydrodynamics under Transient Conditions," Journal of Hydraulic Engineering, Vol. 117, No. 8, pp. 1042-1055.

Henderson, F.M., (1966), Open Channel Flow, Macmillan, New York.

Li, J. and A. McCorquodale, (1999), "Modeling Mixed Flow in Storm Sewers", Journal of Hydraulic Engineering, Vol. 125, No. 11, pp.1170-1180.

NTH Consultants, Ltd. (2000) "Basis of Design Report, Dearborn CSO Control Program," Report to City of Dearborn, September 18, 2000. 